\title{
La biomecánica y psicomotricidad del corredor como factores determinantes para el apoyo del antepie en la carrera
}

\author{
Biomechanics and psychomotricity of the runner as determinant factors for \\ the forefoot support in the race
}

\author{
Andrea Roca Dols ${ }^{1}$, Rubén Sánchez Gómez ${ }^{1,2}$ \\ ${ }^{1}$ Diplomado en Podología. \\ andrea.roca.dols@gmail.com \\ 2 Doctor la Universidad Rey Juan Carlos de Madrid. \\ rusago_@hotmail.com
}

Correspondencia:

Andrea Roca Dols

Camino Motor Serradal, 5

12100, Grao de Castellón. Castellón.

Correo electrónico: andrea.roca.dols@gmail.com

Fecha de recepción: 10 de octubre de 2013

Fecha de aceptación: 19 de julio de 2014

Los autores declaran no tener ningún tipo de interés económico o comercial.

\section{RESUMEN}

Objetivo. Correr descalzo o con calzado minimalista es una nueva tendencia que tiene la finalidad de evitar lesiones en los corredores. Se cree que el calzado deportivo aumenta el número de lesiones al favorece el apoyo de talón respecto al de antepié. Nuestro objetivo es saber si esto es cierto o si es la biomecánica y la psicomotricidad del propio corredor la clave en el mayor o menor apoyo de antepié.

Material y métodos. Se seleccionó a 12 corredores neutros y con ausencia de lesiones a los que se les registraron 3 apoyos de cada pie andando y corriendo en la modalidad de calzados y descalzos mediante la plataforma de presiones digital Podoprint, la cual se situó al $60 \%$ del recorrido $(27 \mathrm{~m})$. Las dos variables estudiadas y comparadas en los contrastes fueron la presión y la superficie.

Resultados. El ir calzado produce cambios en cuanto a presión y superficie en el antepié, la superficie aumenta pero la presión del disminuye. Independientemente de que se corra calzado o descalzo siempre se apoya más el antepié y el apoyo es mayor en el antepié descalzo que en el retropié calzado.

Conclusiones. La biomecánica es la responsable del mayor o menor apoyo de antepié en la carrera, el calzado no condiciona el apoyo de ninguna región del pie pero si modifica la presión y superficie del antepié.

Palabras clave: antepié; presión; lesiones; biomecánica.

\begin{abstract}
Objective. Running barefoot or minimalist footwear is a new trend that is intended to prevent injuries in runners. It is believed that the footwear increases the number of injuries to heel counter favors regarding forefoot. Our goal is to know if this is true or if biomechanics and psychomotor key corridor itself in varying forefoot support.

Material and method. 12 runners were selected neutral and with no injuries to which they are recorded each foot 3 supports walking and running in shoes and barefoot mode by digital pressure Podoprint platform, which amounted to $60 \%$ travel $(27 \mathrm{~m})$. The two variables and contrasts were compared in the pressure and surface.

Results. Going footwear as to produce pressure changes and in the forefoot area, the surface pressure increases but decreases. Regardless of footwear smudging or barefoot always most forefoot support and the support is greater in the forefoot to rearfoot barefoot footwear.

Conclusions. Biomechanics is responsible for more or less support in the race forefoot, the shoe does not condition the support of any region of the foot but if you modify the surface of forefoot pressure.
\end{abstract}

Keywords: forefoot; pressure; injuries; biomechanics.

Sumario: 1. Introducción. 2. Métodos. 3. Resultados. 4. Discusión. 5. Conclusiones. Bibliografía.

Referencia normalizada: Roca Dols, A., Sánchez Gómez, R. La biomecánica y psicomotricidad del corredor como factores determinantes para el apoyo del antepie en la carrera. Rev. Int. Cienc. Podol. 2015; 9(1): 50-62. 


\section{INTRODUCCION}

Según los estudios, El ser humano parece estar especializado para realizar carreras de resistencia por la anatomía y fisiología que presenta en comparación con otros grandes simios. La falta relativa de pelo en el cuerpo, la capacidad excepcional para sudar y almacenar energía de forma elástica en los músculos y tendones del tobillo ${ }^{1,2}$. El consumo aeróbico que manifiesta en las largas distancias, y los dedos del pie más cortos parecen ser adaptaciones para la carrera de resistencia ${ }^{3}$. Además muchos creen que la carrera está en nuestros genes porque el Homo tuvo que correr para alcanzar a la presa antes de la invención de los arcos y las flechas ${ }^{2}$.

Sin embargo, una paradoja que presenta el pie humano es la posición del talón respecto a otros mamíferos. La gran mayoría de mamíferos son digitígrados, su talón se mantiene elevado por encima del suelo, de modo que el animal camina y corre sobre las puntas de sus pies. Se cree que esto mejora la economía del aparato locomotor porque aumenta la longitud del paso. Nuestro talón está situado hacia abajo, somos plantígrados. Es decir, el talón durante la marcha contacta con el suelo y absorbe energía en el inicio de la ejecución del paso; se espera que este mecanismo limite el almacenamiento y la recuperación de energía elástica en los músculos extensores de la articulación del tobillo. Este tipo de apoyo nos hace ser andadores económicos pero no corredores económicos ${ }^{3}$.

Independientemente del porqué empezamos a correr, es evidente que se hizo sin zapatos; el primer ejemplo de calzado se remonta a más de 10.000 años cerca de Fort Rock. Su función era únicamente proteger la planta del $\mathrm{pie}^{4}$. El calzado ha evolucionado mucho hasta el auge de la década de 1970, cuando se inventó el calzado deportivo, el cual tenía incorporado una cantidad significativa de amortiguación y estabilización, comercializado por la comodidad, protección contra lesiones y corrección de los patrones de movimiento ${ }^{4,5}$. La pregunta es ¿por qué se lesionan los corredores a pesar de usarlo? Se sugiere, que las deportivas modernas pueden haber jugado un papel importante en las lesiones, a pesar de que existan otros cambios en nuestras vidas como la alimentación, el medio, la rutina de entrenamiento...
Las características de amortiguación pueden alterar la forma en que se corre y los métodos de control pueden desacondicionar nuestros pies ${ }^{4}$. Los análisis cinéticos y cinemáticos muestran las diferencias biomecánicas en corredores descalzos y calzados. En este estudio se comprobará si existe modificación en la recepción del impacto contra el suelo con y sin zapatillas ${ }^{4,5}$ y si el calzado impone el patrón de apoyo del pie como sugieren algunos artículos ${ }^{6,7}$ además de disminuir la rigidez en las piernas ${ }^{7}$

Sin embargo, en el patrón de pisada parece que influyen otras variables como: las propiedades mecánicas del substrato, la estructura del cuerpo, el entorno de ejecución, la velocidad, el coaching (nivel de formación), la distancia y la frecuencia ${ }^{4,8}$. En este caso vamos a centrarnos en relacionar la pisada con la biomecánica, según la pisada neutra del pie de los corredores para nuestro estudio.

En los pies descalzos o con calzado minimalista aparece una estrategia de adaptación inducida para evitar el apoyo del talón; esta adaptación neural-mecánica consiste en situar el pie en una posición más plana al apoyar que puede mejorar el almacenamiento y restitución de energía elástica a nivel del tobillo y de la musculatura extrínseca e intrínseca del pie. Siendo esto una indicación de fortalecimiento y justificable con la retroalimentación sensorial que experimenta el pie al ir descalzo ${ }^{4,5,9,10,11,12}$. Al correr descalzo la zancada es más corta y más frecuente, por lo tanto el número de impactos es mayor ${ }^{4,7,10}$ pero curiosamente, la absorción de energía total en el miembro inferior es menor $^{13,14}$, el impacto se reduce al correr descal$\mathrm{zo}^{11}$. Según los estudios, incluso en superficies duras las fuerzas de reacción que se generan en el antepié en corredores descalzos son menores que las fuerzas que se generan en el talón en corredores calzados debido a que el mayor cumplimiento de tobillo y la flexión plantar del pie en el momento del impacto, ya que estas adaptaciones disminuyen la masa efectiva del cuerpo que choca contra la suelo ${ }^{5}$. En cambio, en la articulación de la cadera y rodilla disminuye el trabajo ${ }^{13}$.

En el arco longitudinal interno (ALI) también se producen adaptaciones que indican una mayor tensión en él, por el almacenamiento y liberación de energía elástica ${ }^{5,10,12}$. La pierna 
se adapta a la dureza de la superficie manteniéndose rígida, de manera que actúa como un soporte lineal tanto en la carrera como en el salto $^{14}$ Esto justifica como el pie se enfrentaba al impacto del choque antes de la invención de la zapatilla moderna y además sugiere que este mecanismo de apoyo de antepié o mediopié en corredores descalzos podía estar protegiendo los pies y las extremidades inferiores de algunas lesiones que hoy por hoy están experimentando un alto porcentaje de corredores. Ya que la fase más perjudicial de la carrera puede ser el impacto del pie contra el suelo en la fase inicial, porque las fuerzas verticales de reacción del suelo en la fase de contacto de talón se traduce en altos índices de carga ${ }^{4,5}$.

El propósito de este estudio es demostrar que la recepción del impacto contra el suelo en la carrera depende de la biomecánica y forma de correr inherente del corredor teniendo en cuenta como se puede modificar el impacto en función de si el corredor corre calzado o descalzo.

\section{MÉTODOS}

Se han estudiado 12 corredores de media distancia, en las instalaciones del club de atletismo de la localidad de Onda, Castellón. El periodo de selección y la toma de datos duro casi dos meses. Los candidatos fueron 11 hombres y 1 mujer, con edades comprendidas entre 28-

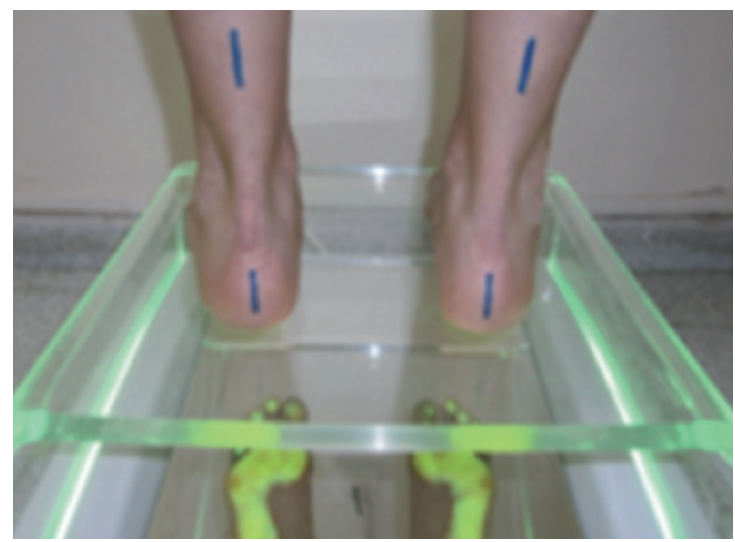

Imagen 1. Corredor neutro sobre el podoscopio, en visión posterior con la línea de Helvin y la línea de valoración del varo tibial trazadas.
55 años, pie neutro (Imagenes 1 y 2) y sin historial de patología en el miembro inferior en los últimos 6 meses; la talla de calzado era la comprendida entre la 42-44 (Tabla 1).

Los criterios de inclusión de los sujetos que muestran la ausencia de patología fueron determinados por los siguientes test: el índice de postura del pie (FPI), el test de windlass, el test de resistencia a la supinación, el doble y simple heel rise test, la huella en el podoscopio y un cuestionario oral histórico sobre lesiones y dolencias. A continuación mostramos una breve explicación de estos test.

- El FPI es una herramienta clínica diagnostica, cuya finalidad es cuantificar el grado de posición neutra, pronada o supinada del pie. Fue descrita por el Dr. Anthony Readmon en $2005^{15,16}$. El objetivo es realizar un método simple de puntuación de 6 factores en la postura del pie por medio de un resultado simple y cuantificable. La valoración se realiza con el paciente en bipedestación con su base de sustentación, apoyo bipodal y ángulo de progresión en estática. Mide y cuantifica la posición del pie en estática y en los tres planos (retropié, mediopié y antepié) y además es una prueba vali$\operatorname{dada}^{17,18}$.

- Los seis criterios clínicos empleados son: palpación de la cabeza del astrágalo, curvatura supra e infra maleolar lateral, posición del calcáneo en el plano

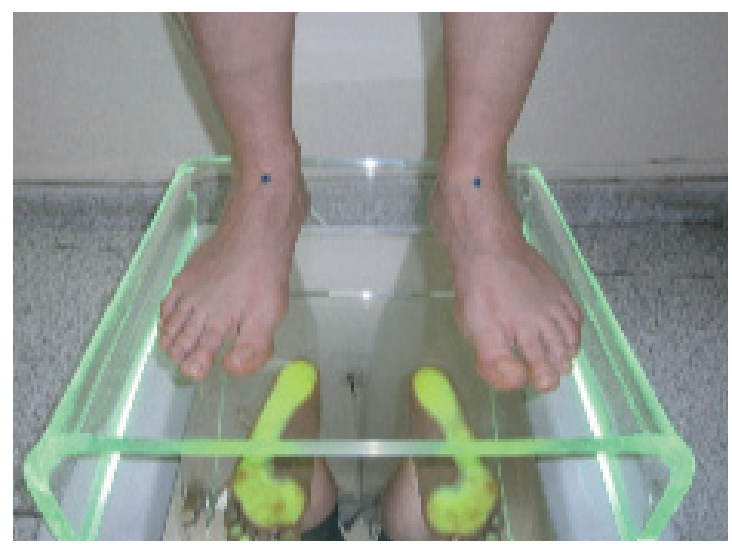

Imagen 2. Corredor neutro sobre el podoscopio, en visión anterior con la orientación del eje de la STJA trazado. 
frontal, prominencia en la región talo navicular, congruencia del arco interno y abducción/aducción del antepié respecto al retropié.

- Los valores de referencia en la puntuación final son:

Normal $=0 \mathrm{a}+5$

Pronado $=+6 \mathrm{a}+9$ / Altamente prona$\mathrm{do}=+10 \mathrm{a}+12$

Supinado $=-1$ a -4 / Altamente supina$\mathrm{do}=-5 \mathrm{a}-12$

- El test de windlass, descrito por Hicks en el año $(1954)^{19}$, valora la integridad de la fascia plantar en pies pronados o planos. Consiste en situar al paciente en bipedestación, con su base de sustentación y su ángulo de progresión en estática. Se le pide al sujeto que no realice ningún tipo de fuerza en el pie. Elevamos el primer dedo desde la falange proximal esperando obtener un aumento del ALI, una rotación externa tibial, un varo de retropié y una planta-flexión del primer radio. Si no vemos estas 4 correcciones calificaremos la maniobra de positiva y estaremos ante un pie con presencia de patología.es decir, la fascia está sometida a mucha tensión y no permite la corrección del pie ${ }^{20}$.

- El test de resistencia a la supinación valora la fuerza necesaria que tiene que ejercer la ortesis para supinar el pie. Se utiliza en pies pronados o planos. Es

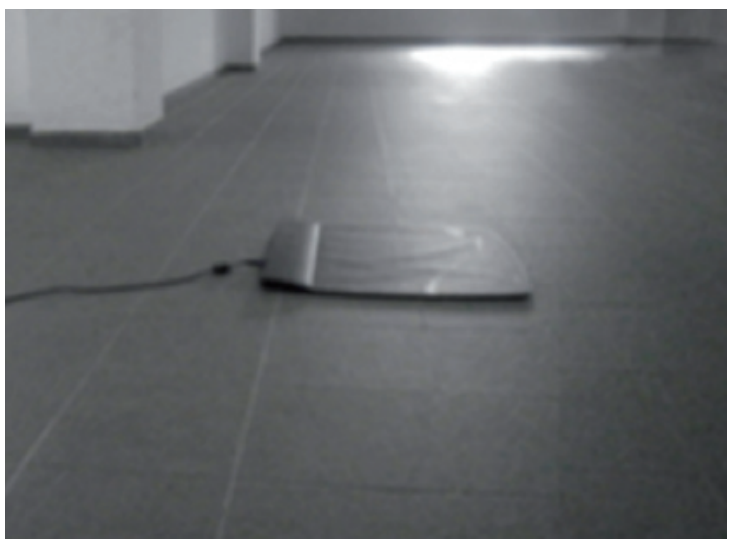

Imagen 3. Plataforma Podoprint situada al $60 \%$ de la distancia a recorrer en las instalaciones del club de atletismo de Onda. una técnica manual que clínicamente puede ser muy útil para prescribir el tratamiento necesario en este tipo de pies. El paciente se sitúa en bipedestación en apoyo bipodal y con su ángulo de progresión en estática. El examinador sitúa el dedo índice y corazón de su mano sobre la zona plantar del escafoides del paciente y intenta desde esta zona supinar el pie. El paciente no debe ni impedir la maniobra ni favorecerla ${ }^{21,22}$.

- El heel rise test valora la integridad del tendón tibial posterior en pies pronados o planos, se le pide al paciente que se ponga de puntillas sobre una pierna (single rise test) o sobre las dos (double rise test). Al ponerse de puntillas sobre una pierna le pedimos que baje el talón hasta el suelo y vuelva a subirlo todo lo que pueda un mínimo de 15 repeticiones, en la momento final de la elevación del talón es cuando trabaja el músculo tibial posterior, es ahí cuando debemos observar si el talón variza o no. En el caso de que no lo haga la maniobra es positiva, es decir hay patología, el tibial no está integro ${ }^{23,24,25}$.

Ninguno de los sujetos dio positivo, es decir, presencia de patología en el pie al realizar las maniobras exploratorias test de windlass y heel rise test, en el caso de la prueba de resistencia a la supinación todos mostraron una resistencia media.

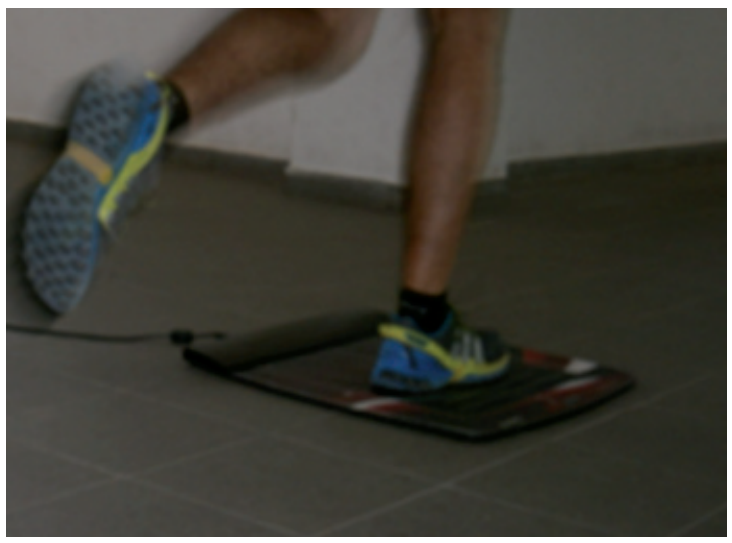

Imagen 4. Impacto del pie sobre la plataforma Podoprint por parte de un corredor neutro con deportiva neutra. 
Inicialmente se les pesó y se les midió; posteriormente se les realizó una valoración más completa de la marcha mediante una plataforma de presiones digital ${ }^{26,27,28}$ (Sistema Podoprint ${ }^{\circledR}$ de la casa Namrol $\left.{ }^{\circledR}\right)$.

La plataforma se situó sobre el suelo de la sala al $60 \%$ de la longitud del camino a recorrer por los participantes ${ }^{5}$, la distancia total de la habitación era de $27 \mathrm{~m}$ para que los participantes pudieran dar un mínimo de 20 zancadas en cada dirección (Imagen 3). El calzado con el que corrieron era el propio de cada sujeto, para asegurar el rendimiento más típico de cada uno de ellos (Imagen 4); la única premisa necesaria es que fuera una deportiva neutra y que tuviera menos de $500 \mathrm{~km}$, para asegurar que el desgaste de la misma no fuera un sesgo añadido a la prueba. Las marcas permitidas en el estudio fueron Asics gel Cumulus ${ }^{\circledR}$, Mizuno Wave Rider ${ }^{\circledR}$, Nike Air Pegaus ${ }^{\circledR}$, Vomero Ready ${ }^{\circledR}$, Adidas Kanadian TRY ${ }^{\circledR}$,New Balance Baddeley ${ }^{\circledR}$, Saucony Jazz ${ }^{\circledR}$. Los sujetos llevaban pantalón corto y camiseta. La velocidad de marcha y de carrera fue la propia de cada uno de ellos, habiendo hecho antes varios ensayos de habituación para llevar una velocidad similar entre todos los corredores y para no modificar la zancada antes de pisar la plataforma. Se registraron 3 apoyos de cada pie en cada condición, dejando un minuto de descanso entre cada medición. Las condiciones fueron:

Andando: los sujetos recorrieron calzados desde el punto de salida 27 metros, realizando la marcha lo más natural posible y pisando en la plataforma sin modificar su marcha. Prime- ro con un pie, y luego con el otro. Se realizaron 5 repeticiones. Posteriormente se repitió esto mismo descalzos.

Corriendo: los sujetos recorrieron calzados desde el punto de salida 27 metros, realizando la marcha lo más natural posible y pisando en la plataforma sin modificar su marcha. Primero con un pie, y luego con el otro. Se realizaron 5 repeticiones. Posteriormente se repitió esto mismo descalzos.

\section{Análisis e interpretación de datos}

Se ha utilizado la estadística descriptiva para valorar las fuerzas reactivas del suelo (FRS) en cada sujeto y en las condiciones mencionadas anteriormente. Utilizando la metodología cuantitativa, hemos valorado la normalidad de la muestra de datos mediante el test de Kolmogorov Smirnov, además de comparar los datos estadísticos mediante la t-student y correlaciones bivariadas para responder a las hipótesis y pregunta de nuestro trabajo.

\section{RESULTADOS}

Con el objetivo de conocer la normalidad de los datos de la muestra se realizó el test de Kolmogorov-Smirnov, con las medias de presión y superficie del apoyo de talón, apoyo completo y despegue de cada pie, tanto andando como corriendo en la modalidad de descalzos y calzados en los 12 corredores. El resultado ha sido en todos los casos un nivel de significación superior a 0,05 , luego podemos decir que los datos siguen una distribución normal.

Tabla 1. Estadísticos de grupo. Valores biométricos y de edad de la muestra total de sujetos del estudio.

\begin{tabular}{lrrrr} 
& Edad & Peso & Altura & $\mathbf{N}^{\mathbf{0}}$ calzado \\
$\mathbf{N}=\mathbf{1 2}$ sujetos & & & & \\
Media & 43 & 79.08 & 176.6 & 43.2 \\
Desviación típica & 6.35 & 10.61 & 6.54 & 0.69 \\
Mínimo & 33 & 61 & 168 & 42 \\
Máximo & 55 & 98.7 & 190 & 44 \\
\hline
\end{tabular}

Edad: medida en años

Peso: medido en $\mathrm{kg}$.

Altura: medida en $\mathrm{cm}$.

$\mathbf{N}^{\mathbf{0}}$ calzado: según la numeración europea

$\mathbf{N}$ : tamaño poblacional de la muestra 
Posteriormente se realizó la prueba de la t de Student con el propósito de conocer si existían diferencias estadísticamente significativas en cuanto a presión y superficie entre el antepié descalzo y el antepié calzado. Tras el contraste de las medias de las 4 combinaciones posibles:

1. Antepié izquierdo andando descalzo (AIAD)-Antepié izquierdo andando calzado (AIAC)

- Presión AIAD con AIAC

Comparando los resultados de la presión en el AIAD $(2174,83 \pm 242,36)$ y en el AIAC $(1445,97 \pm 139,91)$ se encontraron diferencias estadísticamente significativas $(\mathrm{p}<0,05)$ de forma que al ir descalzo aumentó la presión en el antepié izquierdo.

- Superficie del AIAD con AIAC Comparando los resultados de superficie en el AIAD $(59,22 \pm 3,24)$ y en el AIAC $(67,50 \pm 6,14)$ se encontraron diferencias estadísticamente significativas $(p<0,05)$ de forma que al ir calzado aumentó la superficie de apoyo en el antepié.

2. Antepié derecho andando descalzo (ADAD)-Antepié derecho andando calzado (ADAC)

\section{- Presión ADAD con ADAC}

Comparando los resultados de la presión en el ADAD $(2269,47 \pm 246,43)$ y en el ADAC $(1484,39 \pm 126,97)$ se encontraron diferencias estadísticamente significativas $(\mathrm{p}<0,05)$ de forma que al ir descalzo aumento la presión en el antepié.

- Superficie del ADAD con ADAC

Comparando los resultados de superficie en el $\operatorname{ADAD}(58,89 \pm 2,90)$ y en el ADAC $(72,31 \pm 4,89)$ se encontraron diferencias estadísticamente significativas $(\mathrm{p}<0,05)$ de forma que al ir calzado aumento la superficie de apoyo en el antepié.

3. Antepié izquierdo corriendo descalzo (AICD)-Antepié izquierdo corriendo cazado (AICC)

- Presión AICD con AICC

Comparando los resultados de la presión en el AICD $(2047,31 \pm 263,09)$ y en el AICC $(1500,39 \pm 147,7)$ se encontraron diferencias estadísticamente significativas $(\mathrm{p}<0,05)$ de forma que al ir descalzo aumentó la presión en el antepié.

- Superficie del AICD con AICC
Comparando los resultados de superficie en el AICD $(58,89 \pm 5,96)$ y en el AICC $(72,31 \pm 4,89)$ se encontraron diferencias estadísticamente significativas $(\mathrm{p}<0,05)$ de forma que al ir calzado aumento la superficie.

4. Antepié derecho corriendo descalzo (ADCD)-Antepié derecho corriendo calzado (ADCC)

- Presión ADCD con ADCC

Comparando los resultados de presión en el ADCD $(2172,08 \pm 268,43)$ y en el ADCC $(1482,17 \pm 136,40)$ se encontraron diferencias estadísticamente significativas $(\mathrm{p}<0,05)$ de forma que al ir descalzo aumento la presión en el antepié.

- Superficie del ADCD con ADCC

Comparando los resultados de superficie en el ADCD $(57,75 \pm 3,50)$ y en el ADCC $(69,3 \pm 6,39)$ se encontraron diferencias estadísticamente significativas $(p<0,05)$ de forma que al ir calzado aumento la superficie de apoyo en el antepié.

Por tanto se encontró que las diferencias eran estadísticamente significativas $(p<0,05)$ tanto en la variable de presión como en la de superficie. Siendo la presión mayor en el antepié descalzo y la superficie menor en el antepié descalzo (Tablas 2 y 3 ).

A continuación se realizó de nuevo la prueba de la t de Student para contrastar los apoyos de retopié en las diferentes condiciones, con el propósito de conocer si existían diferencias estadísticamente significativas en cuanto a presión y superficie entre el retropié descalzo y el retropié calzado:

1. Retropié izquierdo andando descalzo (RIAD)-Retropié izquierdo andando calzado (RIAC)

- Presión RIAD con RIAC

Comparando los resultados de la presión en el RIAD $(1660,39 \mathrm{gr} / \mathrm{cm} \pm 150,89) \mathrm{y}$ en el RIAC $(1408,92 \pm 166,30)$ no se encontraron diferencias estadísticamente significativas $(\mathrm{p}>0,05)$.

- Superficie del RIAD con RIAC

Comparando los resultados de superficie en el RIAD $(33,86 \pm 3,10)$ y en el RIAC $(48,97 \pm 4,12)$ se encontraron diferencias estadísticamente significativas $(p<0,05)$ 


\begin{tabular}{|c|cc|c|ccc|} 
CORRIENDO & $\begin{array}{c}\text { DESCALZO } \\
\text { presión }\end{array}$ & $\begin{array}{c}\text { CALZADO } \\
\text { presión }\end{array}$ & p_valor & $\begin{array}{c}\text { DESCALZO } \\
\text { superficie }\end{array}$ & $\begin{array}{c}\text { CALZADO } \\
\text { superficie }\end{array}$ & p_valor \\
\hline antepié izq & $2047,31 \pm 263,09$ & $1500 \pm 147,7$ & $\mathbf{0 , 0 0 0}$ & $58,25 \pm 5,96$ & $68,61 \pm 7,13$ & $\mathbf{0 , 0 0 4}$ \\
\hline antepié dcho & $2172,08 \pm 268,43$ & $482,17 \pm 136,40$ & $\mathbf{0 , 0 0 0}$ & $57,75 \pm 3,50$ & $69,3 \pm 6,39$ & $\mathbf{0 , 0 0 0}$ \\
\hline ANDANDO & & & p_valor & & & p_valor \\
\hline antepié izq & $2174,83 \pm 242,36$ & $1445,97 \pm 139,91$ & $\mathbf{0 , 0 0 0}$ & $59,22 \pm 3,24$ & $67,50 \pm 6,14$ & $\mathbf{0 , 0 2 5}$ \\
\hline antepié dcho & $2269,47 \pm 246,43$ & $1484,39 \pm 126,97$ & $\mathbf{0 , 0 0 0}$ & $58,89 \pm 2,90$ & $72,31 \pm 4,89$ & $\mathbf{0 , 0 0 0}$ \\
\hline
\end{tabular}

Tabla 2. Contraste de medias de presión y superficie en el antepié corriendo y andando en las dos condiciones estudiadas, descalzo y calzado. Se consideran resultados estadísticamente significativos cuando la $\mathrm{p}<0,05$, con unas diferencias estadísticamente muy significativas con la $\mathrm{p}<0,001$. El significado de las abreviaturas empleadas en la tabla es la siguiente: izq $=$ izquierdo y dcho $=$ derecho. La unidad de presión es el $\mathrm{gr} / \mathrm{cm}^{2}$ y la de superficie el $\mathrm{cm}^{2}$.

\begin{tabular}{lllll} 
ANDANDO & $\begin{array}{l}\text { Presión antepié } \\
\text { derecho }\end{array}$ & $\begin{array}{l}\text { Presión antepié } \\
\text { izquierdo }\end{array}$ & $\begin{array}{l}\text { Superficie antepié } \\
\text { derecho }\end{array}$ & $\begin{array}{l}\text { Superficie antepié } \\
\text { izquierdo }\end{array}$ \\
\hline Descalzo respecto Calzado & Disminuye & Disminuye & Aumenta & Aumenta \\
\hline CORRIENDO & & & & Aumenta \\
\hline Descalzo respecto Calzado & Disminuye & Disminuye & Aumenta & Aumenta \\
\hline
\end{tabular}

Tabla 3. Comparativa de la media de las medias de presión y superficie en el antepié, entre descalzo y calzado, en las dos situaciones diferentes de andar y correr. El significado de las abreviaturas empleadas en la tabla es la siguiente: $\mathrm{izq}=$ izquierdo $\mathrm{y} \mathrm{dcho}=$ derecho. La unidad de presión es el gr/ $\mathrm{cm}^{2}$ y la de superficie el $\mathrm{cm}^{2}$.

\begin{tabular}{|c|cc|cccc} 
CORRIENDO & $\begin{array}{c}\text { DESCALZO } \\
\text { presión }\end{array}$ & $\begin{array}{c}\text { CALZADO } \\
\text { presión }\end{array}$ & p_valor & $\begin{array}{c}\text { DESCALZO } \\
\text { superficie }\end{array}$ & $\begin{array}{c}\text { CALZADO } \\
\text { superficie }\end{array}$ & p_valor \\
\hline retropié izq & $1008,36 \pm 133,68$ & $960,25 \pm 138,32$ & $\mathbf{6 , 1 0}$ & $27,39 \pm 4,43$ & $38,14 \pm 7,76$ & $\mathbf{0 , 0 0 2}$ \\
retropié dcho & $973,03 \pm 149,58$ & $1037,69 \pm 127,42$ & $\mathbf{0 , 3 9 4}$ & $28,14 \pm 5,32$ & $39,69 \pm 9,64$ & $\mathbf{0 , 0 0 4}$ \\
\hline ANDANDO & & & p_valor & & & p_valor \\
\hline retropié izq & $1660,39 \pm 150,89$ & $1408,92 \pm 166,30$ & $\mathbf{0 , 5 2}$ & $33,86 \pm 3,10$ & $48,97 \pm 4,12$ & $\mathbf{0 , 0 0 1}$ \\
\hline retropié dcho & $1593,28 \pm 176,69$ & $1505,25 \pm 162,43$ & $\mathbf{0 , 3 4 0}$ & $31,78 \pm 2,59$ & $50,11 \pm 4,65$ & $\mathbf{0 , 0 0 0}$ \\
\hline
\end{tabular}

Tabla 4. Contraste de medias de presión y superficie en el retropié corriendo y andando en las dos condiciones estudiadas, descalzo y calzado. Se consideran resultados estadísticamente significativos cuando la $\mathrm{p}<0,05$, con unas diferencias estadísticamente muy significativas con la $\mathrm{p}<0,001$. El significado de las abreviaturas empleadas en la tabla es la siguiente: $\mathrm{izq}=$ izquierdo y dcho= derecho. La unidad de presión es el $\mathrm{gr} / \mathrm{cm}^{2}$ y la de superficie el $\mathrm{cm}^{2}$. 


\begin{tabular}{lllll} 
ANDANDO & $\begin{array}{l}\text { Presión retropié } \\
\text { derecho }\end{array}$ & $\begin{array}{l}\text { Presión retropié } \\
\text { izquierdo }\end{array}$ & $\begin{array}{l}\text { Superficie } \\
\text { retropié derechoo }\end{array}$ & $\begin{array}{l}\text { Superficie } \\
\text { retropié izquierdo }\end{array}$ \\
\hline $\begin{array}{l}\text { Descalzo respecto Calzado } \\
\text { CORRIENDO }\end{array}$ & Disminuye & Disminuye & Aumenta & Aumenta \\
\hline Descalzo respecto Calzado & Aumenta & Disminuye & Aumenta & Aumenta \\
\hline
\end{tabular}

Tabla 5. Comparativa de la media de las medias de presión y superficie en el retropié, entre descalzo y calzado, en las dos situaciones diferentes de andar y correr. El significado de las abreviaturas empleadas en la tabla es la siguiente: izq=izquierdo y dcho= derecho. La unidad de presión es el $\mathrm{gr} / \mathrm{cm}^{2}$ y la de superficie $\mathrm{el} \mathrm{cm}^{2}$.

de forma que al ir calzado aumentó la superficie de apoyo en el retropié.

2. Retropié derecho andando descalzo (RDAD)-Retropié derecho andando calzado (RDAC)

\section{- Presión RDAD con RDAC}

Comparando los resultados de la presión en el RDAD $(1593,28 \mathrm{gr} / \mathrm{cm} \pm 176,69)$ $y$ en el RDAC $(1505,25 \pm 162,43)$ no se encontraron diferencias estadísticamente significativas $(p>0,05)$.

- Superficie del RDAD con RDAC

Comparando los resultados de superficie en el RDAD $(31,78 \pm 2,59)$ y en el RDAC $(50,11 \pm 4,65)$ se encontraron diferencias estadísticamente significativas $(\mathrm{p}<0,05)$ de forma que al ir calzado aumento la superficie de apoyo en el retropié.

3. Retropié izquierdo corriendo descalzo (RICD)-Retropié izquierdo corriendo cazado (RICC)

\section{- Presión RICD con RICC}

Comparando los resultados de la presión en el RICD $(1008,36 \mathrm{gr} / \mathrm{cm} \pm 133,68)$ y en el RICC $(925,25 \pm 138,32)$ no se encontraron diferencias estadísticamente significativas $(\mathrm{p}>0,05)$.

- Superficie del RICD con RICC

Comparando los resultados de superficie en el RICD $(27,39 \pm 4,43)$ y en el RICC $(38,14 \pm 7,76)$ se encontraron diferencias estadísticamente significativas $(\mathrm{p}<0,05)$ de forma que al ir calzado aumento la superficie en el retropié.

4. Retropié derecho corriendo descalzo (RDCD)-Retropié derecho corriendo calzado (RDCC)

- Presión RDCD con RDCC
Comparando los resultados de presión en el RDCD $(973,03 \pm 49,58)$ y en el RDCC $(1037,69 \pm 127,42)$ no se encontraron diferencias estadísticamente significativas $(\mathrm{p}>0,05)$.

- Superficie del RDCD con RDCC

Comparando los resultados de superficie en el RDCD $(28,14 \pm 5,32)$ y en el RDCC $(39,69 \pm 9,64)$ se encontraron diferencias estadísticamente significativas $(\mathrm{p}<0,05)$ de forma que al ir calzado aumento la superficie de apoyo en el retropié.

Los resultados muestran diferencias significativas en cuanto a superficie pero no significativas en cuanto a presión al comprar el retropié calzado y descalzo en las dos modalidades estudiadas corriendo y andando (Tablas 4 y 5).

Además se valoró si el apoyo de antepié es mayor que el apoyo de retropié al correr descalzo y si el apoyo de retropié es mayor que el de antepié al correr calzado, para ello se compararon dos condiciones por premisa:

1. Antepié izquierdo corriendo descalzo (AICD)-Retropié izquierdo corriendo descalzo(RICD)

\section{- Presión AICD con RICD}

Comparando los resultados de presión en el AICD y en el RICD se encontraron diferencias estadísticamente significativas $(\mathrm{p}<0,05)$, el apoyo de antepié $(2047,31 \pm 263,09)$ y el apoyo de retropié $(1008,36 \pm 133,68)$, de forma que la presión del antepié es mayor a la del retropié al correr descalzo.

\section{- Superficie del AICD con RICD}

Comparando los resultados de la superficie de apoyo en el AICD y en el RICD 
se encontraron diferencias estadísticamente significativas $(\mathrm{p}<0,05)$, el apoyo de antepié $(58,25 \pm 5,96)$ y el apoyo de retropié $(27,39 \pm 4,43)$, de forma que la superficie del antepié es mayor a la del retropié al correr descalzo.

2. Antepié derecho corriendo descalzo (ADCD)-Retropie derecho corriendo descalzo(RDCD)

- Presión ADCD con RDCD

Comparando los resultados de presión en el ADCD y en el RDCD se encontraron diferencias estadísticamente significativas $(\mathrm{p}<0,05)$, el apoyo de antepié $(2172,08 \pm 268,43)$ y el apoyo de retropié $(973,03 \pm 149,48)$, de forma que la presión del antepié es mayor a la del retropié al correr descalzo.

- Superficie del ADCD con RDCD

Comparando los resultados de la superficie de apoyo en el ADCD y en el RDCD se encontraron diferencias estadísticamente significativas $(\mathrm{p}<0,05)$, el apoyo de antepié $(57,75 \pm 3,50)$ y el apoyo de retropié $(28,14 \pm 5,32)$, de forma que la superficie del antepié es mayor a la del retropié al correr descalzo.

3. Antepié izquierdo corriendo calzado (AICC)-Retropié izquierdo corriendo calzado(RICC)

- Presión AICC con RICC

Comparando los resultados de presión en el AICC y en el RICC se encontra- ron diferencias estadísticamente significativas $(\mathrm{p}<0,05)$, el apoyo de antepié $(1500 \pm 147,7)$ y el apoyo de retropié $(960,25 \pm 138,32)$, de forma que la presión del antepié es mayor a la del retropié al correr calzado

- Superficie del AICC con RICC

Comparando los resultados de la superficie de apoyo en el AICC y en el RICC se encontraron diferencias estadísticamente significativas $(p<0,05)$, el apoyo de antepié $(68,61 \pm 7,13)$ y el apoyo de retropié $(38,14 \pm 7,76)$, de forma que la superficie del antepié es mayor a la del retropié al correr calzado.

4. Antepié derecho corriendo calzado (ADCC)-Retropié derecho corriendo calzado(RDCC)

- Presión ADCC con RDCC

Comparando los resultados de presión en el ADCC y en el RDCC se encontraron diferencias estadísticamente significativas $(\mathrm{p}<0,05)$, el apoyo de antepié $(1482,17 \pm 136,40)$ y el apoyo de retropié $(1037,69 \pm 127,42)$, de forma que la presión del antepié es mayor a la del retropié al correr calzado

- Superficie del ADCC con RDCC

Comparando los resultados de la superficie de apoyo en el ADCC y en el RDCC se encontraron diferencias estadísticamente significativas $(\mathrm{p}<0,05)$, el apoyo de antepié $(69,3 \pm 6,39)$ y el apoyo

\begin{tabular}{c|cccccc}
\hline CORRIENDO & antepié izq & retropié izq & p_valor & antepié dcho & retropié dcho & p_valor \\
$\begin{array}{c}\text { DESCALZO } \\
\text { presión }\end{array}$ & $2047,31 \pm 263,09$ & $1008,36 \pm 133,68$ & $\mathbf{0 , 0 0 0}$ & $2172 \pm 268,43$ & $973,02 \pm 149,48$ & $\mathbf{0 , 0 0 0}$ \\
$\begin{array}{c}\text { DESCALZO } \\
\text { superficie }\end{array}$ & $58,25 \pm 5,96$ & $27,39 \pm 4,43$ & $\mathbf{0 , 0 0 0}$ & $57,75 \pm 3,50$ & $28,14 \pm 5,32$ & $\mathbf{0 , 0 0 0}$ \\
$\begin{array}{c}\text { CALZADO } \\
\text { presión }\end{array}$ & $1500 \pm 147,7$ & $960,25 \pm 138,32$ & $\mathbf{0 , 0 0 0}$ & $1482,17 \pm 136,40$ & $1037,69 \pm 127,42$ & $\mathbf{0 , 0 0 0}$ \\
$\begin{array}{c}\text { CALZADO } \\
\text { superficie }\end{array}$ & $68,61 \pm 7,13$ & $38,14 \pm 7,76$ & $\mathbf{0 , 0 0 0}$ & $69,30 \pm 6,39$ & $39,69 \pm 9,64$ & $\mathbf{0 , 0 0 0}$ \\
\hline
\end{tabular}

Tabla 6. Contraste de medias de presión y superficie en el antepié y retropié corriendo y andando en las dos condiciones estudiadas, descalzo y calzado. Se consideran resultados estadísticamente significativos cuando la $p<0,05$, con unas diferencias estadísticamente muy significativas con la $p<0,001$. El significado de las abreviaturas empleadas en la tabla es la siguiente: izq=izquierdo y dcho= derecho. La unidad de presión es $\mathrm{el} \mathrm{gr} / \mathrm{cm}^{2}$ y la de superficie el $\mathrm{cm}^{2}$. 


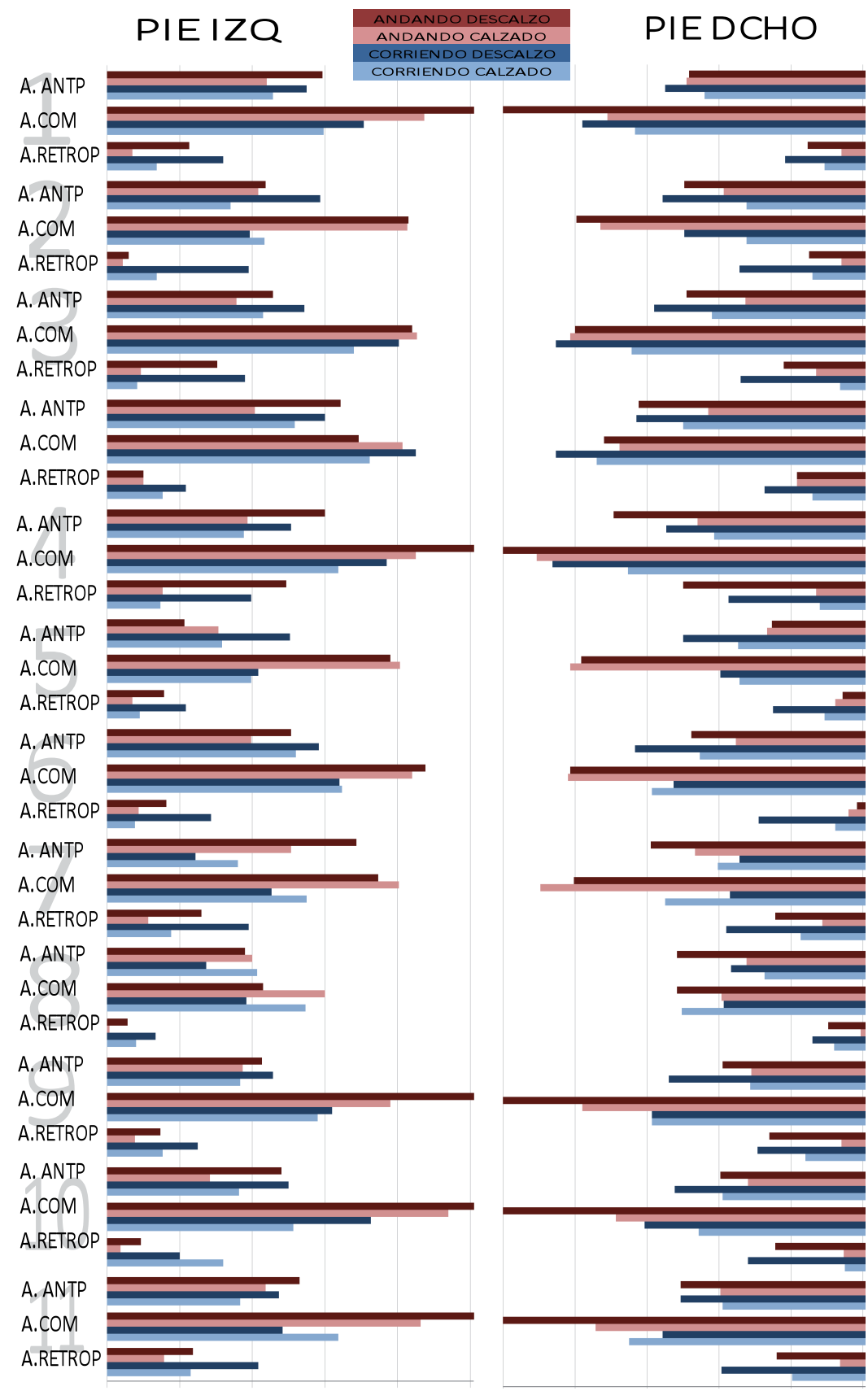

Figura 1. Comparación de las medias de superficie en las 3 fases de la marcha en cada sujeto en ambos pies. En la condición de calzado, descalzo, corriendo y andando (A.PIE) despegue, (A.COM) apoyo completo, (R.PIE) apoyo de talón.

Leyenda de la Figura 1:

Eje X: Área de superficie, cuya unidad de medida es el $\mathrm{cm}^{2}$

Eje Y: Fases de la marcha estudiadas en cada corredor

A.ANTP: Apoyo de antepié

A.COM: Apoyo completo

A.RETROP: Apoyo de retropié 
de retropié $(39,69 \pm 9,64)$, de forma que la superficie del antepié es mayor a la del retropié al correr calzado.

Con estos resultados indicamos que el apoyo de antepié es superior al de retropié al correr sin importar la condición de descalzo o calzado siendo las diferencias significativas (Tabla 6).

\section{DISCUSIÓN}

Según el análisis de los datos obtenidos, la biomecánica y la psicomotricidad del corredor influyen en el mayor o menor apoyo del antepié independientemente de si corre calzado o descalzo. A pesar de que la primera parte de los resultados de nuestro estudio muestran cómo las diferencias de presión y superficie en la región del antepié son muy diferentes al correr calzado y descalzo, la segunda parte de los resultados indican que el apoyo de antepié es superior al de retropié al correr en cuanto a presión y superficie, sin importar la condición de descalzo o calzado.

Por ello este estudio pone en duda la idea principal de algunos de los artículos de nuestra revisión bibliográfica, los cuales afirman que existen cambios en la región de apoyo del pie en función de la condición en la que se corra. Estos artículos sugieren que al correr descalzo el pie se sitúa en una posición más plana al aterrizar ${ }^{11}$ lo que provoca una mayor flexión plantar del tobillo y por tanto el apoyo del antepié 4,5, $, 13,1,29$. Además dos de estos artículos mantienen la hipótesis de que el calzado deportivo fomenta el apoyo de talón por el acolchado presente en la zona del retropié, el cual favorece la flexión dorsal del tobillo y consecuentemente el apoyo de talón ${ }^{4,5}$.

Este patrón de apoyo de antepié que se atribuye al correr descalzo se describe como una estrategia de adaptación inducida del propio organismo para evitar el apoyo de talón, porque así se evita tensión mecánica y fuerza de impacto en la región del talón, la cual puede estar creando en numerosos corredores lesiones como: fascitis plantar, síndrome de estrés tibial, molestias de rodilla... ${ }^{4,5,7,13,1,29}$ Junto con estas ideas hemos tenido en cuenta las conclusiones de otro de nuestros artículos el cual informa de que tras un cuestionario de relaciones entre el tipo de calzado, patrón de pisada $\mathrm{y}$ incidencia de lesiones en los distintos corredores, los corredores calzados son más propensos a padecer lesiones en las extremidades inferiores que los corredores que usan calzado minimalista ${ }^{29}$.

Tras esta información hemos hecho varios contrastes para comprobar si todo lo explicado anteriormente es cierto:

\section{Presión y superficie en el antepié des- calzo y calzado, corriendo y andando}

Calzado siempre hay más superficie de apoyo que descalzo y de manera muy significativa $(p<0,05)$ independientemente de la fase de la marcha y del área del pie estudiada. El antepié derecho pasa de una superficie al correr descalzo de $(57,75 \pm 3,50)$ a $(69,3 \pm 6,39)$ al correr calzado (Tablas 2, 3 y 5). Al correr siempre hay menos superficie que al andar, (excepto en el antepié izquierdo, que es por muy poco y puede ser achacable a una pequeña variación de sesgo asociada a la posición del pie en ese momento de apoyo) dado que al aumentar la velocidad de la marcha el pie apoya menos tiempo en el suelo (Tabla 2).

Descalzo siempre hay más presión $(2047,31 \pm 263,09)$ que al ir calzado $(1500 \pm 147,7)$, ejemplo del antepié izquierdo al correr. Vemos como la presión varia de forma muy significativa $(p<0,001)$ (Tabla 2). Cabe destacar que la presión es mayor al andar que al correr descalzo.

\section{Presión y superficie en el antepié des- calzo y en el retropié descalzo, corriendo}

Ambas variables son mayores siempre en el antepié, siendo las diferencias muy significativas $(\mathrm{p}<0,0001)$ en ambos casos, $(2047,31 \pm 263,09)$ presión del antepié izquierdo corriendo $(1008,36 \pm 133,68)$ presión del retropié izquierdo corriendo; superficie antepié izquierdo corriendo $(58,25 \pm 5,96)$ superficie de retropié izquierdo corriendo $(27,39 \pm 4,43)$ (Tabla 6). Independientemente de la fase de la marcha e independientemente de si se está corriendo o andando, el antepié siempre va a tener más superficie de apoyo que el retropié. Estos resultados concuerdan con la idea de que el correr descalzo favorece el apoyo de antepié.

3. Presión y superficie en el antepié descalzo y en el retropié calzado, corriendo: 
Ambas variables son mayores siempre en el antepié con respecto al retropié, presión del antepié derecho descalzo $(2172 \pm 268,43)$, presión retropié derecho calzado $(1037,69 \pm 127,42)$; superficie antepié derecho descalzo $(57,75 \pm 3,50)$, superficie retropié derecho calzado $(39,69 \pm 9,64)$ siendo las diferencias muy significativas $(p<0,001)$ (Tabla 6). Estos resultados discrepan de los de los estudios mencionados anteriormente porque estos afirman que el calzado deportivo aumenta el apoyo de talón y consecuentemente el impacto en esta región pudiendo ser la causa de numerosas lesiones.

Uno de los artículos revisados mantiene la idea de que el tipo de pie se asocia con la mecánica de aterrizaje y que por ello pueden subyacer lesiones en las extremidades inferiores ${ }^{30}$. De manera que esta conclusión está en concordancia con nuestros hallazgos en el aspecto de que es la propia biomecánica del individuo la que condiciona el apoyo de antepié o retropié, y que por tanto, correr calzado no tiene porque ser la causa del aumento de lesiones relacionadas con el impacto anterior o posterior en algunos deportistas.

No obstante se necesitan más estudios retrospectivos sobre esta nueva tendencia de correr descalzo o con calzado minimalista y la realización de estudios más amplios que contemplen variables tales como la velocidad de carrera, nivel de formación, la capacidad del individuo en función de su anatomía (muchas veces el nivel de exigencia propio puede ser desmesurado de acuerdo a sus posibilidades), distancia y frecuencia. Así como estudios que valoren la cinemática y las presiones en una muestra más amplia de sujetos con un mismo calzado, con el fin de justificar muchas de las lesiones de este colectivo y poder así prevenirlas.
Por último comentar que es importante educar con seguridad a los corredores, con el fin de evitar lesiones que pueden darse por un cambio repentino de una modalidad a la otra o por un aterrizaje brusco de antepié. Teniendo en cuenta que según nuestro estudio no hay justificación para que ningún tipo de corredor (ni con lesiones ni sin lesiones) realice una transición a este nuevo estilo de correr, porque según nuestros datos da igual correr calzado que descalzo, porque el apoyo de antepié es predominante siempre. Además de que no se sabe cómo puede afectarles el correr descalzo o con calzado minimalista a los corredores con afecciones especiales como diabetes, hallux abductus valgus o neuromas.

\section{CONCLUSIONES}

- La biomecánica y la psicomotricidad del corredor son las responsables del mayor o menor apoyo de antepié en la carrera.

- El calzado no condiciona el apoyo de talón, con lo cual no podemos justificar con él determinadas lesiones deportivas porque independientemente de correr calzado o descalzo siempre se apoya más el antepié.

- El calzado deportivo si esta relacionado con la recepción del impacto del pie con el suelo en el antepié porque varía la presión y la superficie:

- Al andar y correr calzado siempre hay más superficie de apoyo que al andar y correr descalzo.

- Al andar y correr calzado siempre hay menos presión que al andar y correr descalzo, siendo la presión mayor al andar descalzo que al correr descalzo.

\section{BIBLIOGRAFÍA}

1. Bramble DM, Liberman DE. Endurance running and the evolution of Homo. Nature. 2004; 432:34552. Doi:10.1371/journal.pone.0000418.

2. Carrier DR. The energetic paradox of human running and hominid evolution. Curr Anthropol.1984; 25:483-95. Doi: 10.1186/1471-2148-8-273.

3. Cunningham CB, Schilling N, Anders C, Carrier DR. The influence of foot posture on the cost of transport in humans. J Exp Biol.2010; 213(5):790-7. doi:10.1242/jeb.038984.

4. AR. Davis IS. Barefoot Running: Biomechanics and Implications for Running Injuries. Curr Sports Med Rep. 2012; 244-50. Doi:10,1249/JSR.0b013e31826c9bb9. 
5. Lieberman DE, Venkadesan M, Wrbel WA, Dauod Al, D' Andrea S, Davis IS, Mang'eni RO, Pitsiladis Y. Foot strike patterns and collision forces in habitually barefoot versus shod runners. Nature.2010; 463(7280):531-5. Doi: 10.1038/nature08723.

6. Morico C, Lake MJ, Gueguen N, Rao G, Baly L. Y Biomech. The influence of footwear on foot motion during walking and running. 2009; 42 (13):2081-8. Doi:10.106/j.jbiomech.2009.06.015.

7. Squadrone R, Gallozzi C.Biomechanical and physiological comparison of barefoot and the shod conditions in experienced barefoot runners. Nature.2010;463(7280):531-5.

8. Hatala KG, Dingwall HL, Wunderlich RE, Richmond BG. Variation in foot strike patterns during running among habitually barefoot populations. Plos One. 2013; 8(1). Doi:10.137/journal.

9. De Wit B, De Clercq D, Aerts. Biomechanical analysis of the stance phase during barefoot and shod running. P.J Biomech.2000; 33(3):269-78.

10. Perl DP, Daoud Al, Lieberman DE. Effects of footwear and strike type on running economy. Med Sci Sports Exerc. 2012;44 (7): 1335-43. Doi: 10.1249/MSS.0B013E318247989e.

11. Divert C, Mornieux G, Baur H, Mayer F, Belli A. Mechanical comparison of barefoot and shod running. Int J Sports Med. 2005;26(7):593-8.

12. Robbins SE, Hanna AM. Running-related injury prevention through barefoot adaptions. Med Sci Sports Exerc.1987;19(2):148-56

13. Williams DS 3rd, Green DH, Wurzinger B. Changes in lower extremity movement and power absorption during forefoot striking and barefoot running. Int J Sports Phys Ther.2012; 7(5):525-32.

14. Bishop M, Fiolkowski P, Conrad B, Brunt D, Horodyski M. Athletic footwear, leg stillness, and running Kinematics. J Athl Train.2006;41(4):387-92.

15. Sánchez-Rodríguez R, Martínez-Nova A, Escamilla-Martínez E, Pedrera-Zamorano JD. Can the Foot Posture Index or their individual criteria predict dynamic plantar pressures? Gait Posture. 2012;36(3):591-5. Doi: 10.1016/j.gaitpost.2012.05.024.

16. Redmond AC, Crane YZ, Menz HB. Normative values for the Foot Posture Index. J Foot Ankle Res. 2008;1(1):6. Doi: 10.1186/1757-1146-1-6.

17. Redmond AC, Crosbie J, Ouvrier RA. Development and validation of a novel rating system for scoring standing foot posture: the Foot Posture Index. Clin Biomech.2006; 21(1):89-98. Doi:10.1016/j.clinbiomech.2005.08.002

18. Keenan AM, Redmond AC, Horton M, Conaghan PG, Tennant A. The Foot Posture Index: Rasch analysis of a novel, foot-specific outcome measure. Arch Phys Med Rehabil. 2007; 88(1):88-93.

19. Hicks JH.Mechanics of the foot: part II. Plantar aponeurosis and the arch. J Anat 1954;88(1): 25-30.

20. De Garceau D, Dean D, Requejo SM, Thordarson DB. The association between diagnosis of plantar fasciitis and Windlass test results. Foot Ankle Int. 2003;24(3):251-5.

21. Griffiths IB, McEwan IM. Reliability of a new supination resistance measurement device and validation of the manual supination resistance test. J Am Podiatr Med Assoc. 2012;102(4):278-89.

22. Noakes H, Payne C. The reliability of the manual supination resistance test. J Am Podiatr Med Assoc. 2003;93(3):185-9.

23. Yocum A, McCoy SW, Bjornson KF, Mullens P, Burton GN. Reliability and validity of the standing heel rise test. Phys Occup Ther Pediatr. 2010;30(3):190-204. Doi: 10.3109/01942631003761380

24. Olsson N, Karlsson J, Eriksson BI, Brorsson A, Lundberg M, Silbernagel KG. Ability to perform a single heel-rise is significantly related to patient-reported outcome after Achilles tendon rupture. J Med Sci Sports. 2012.

25. Silbernagel KG, Steele R, Manal K. Deficits in heel-rise height and achilles tendon elongation occur in patients recovering from an Achilles tendon rupture. Am J Sports Med. 2012;40(7):1564-71. Doi: 10.1111/j.1600-0838.2012.01497.

26. Hafer JF, Lenhoff MW, Song J, Jordan JM, Hannan MT, Hillstrom HJ. Reliability of plantar pressure platforms. Gait Posture. 2013. doi: 10.1016/j.gaitpost.2013.01.028.

27. Razak AH, Zayegh A, Begg RK, Wahab Y. Foot plantar pressure measurement system: a review. Sensors. 2012;12(7):9884-912. Doi: 10.3390/s130303527.

28. Ramachandra P, Maiya AG, Kumar P. Test-retest reliability of the Win-Track analyzing the gait parameters and plantar pressures during barefoot walking in healthy adults. Foot Ankle Spec. 2012;5(5):306-12.

29. Goss DL, Gross MT. Relationships among self-reported shoe type, footstrike pattern, and injury incidence. US Army Med Dep J. 2012;25-30.

30. Powell H, Hanson N, Long B, Williams D. Frontal plane landing mechanics in high arched compared with low-arched female Athletes. Clin J Sport Med. 2012; 8:430-5. 